\section{Recontacting in clinical genetics and genomic medicine? We need to talk about it}

European Journal of Human Genetics (2017) 25, 520-521; doi:10.1038/ejhg.2017.8; published online 8 February 2017

\section{THE PROBLEM}

An editorial in the $A J M G,{ }^{1}$ which accompanied the first systematic review conducted on the topic, ${ }^{2}$ suggested that advances in nextgeneration sequencing technologies and the volume of genomic information they deliver are leading clinicians to consider recontacting former patients. Examples might be: (a) to discuss whether more sensitive testing could now provide a diagnosis or health prediction, which had not previously been possible; (b) to review the evidence for particular genetic findings playing a part in the patient's disease; or (c) to revise the interpretation of a genetic variant previously identified. As the Otten et al. ${ }^{2}$ systematic review shows, there is a perceived need for guidance in this area. However, it is not clear whether a standard protocol or consensus guidelines are necessary, or indeed whether a duty to recontact exists at all.

\section{OUR PROJECT}

We conducted a survey of UK Regional Clinical Genetics Services, ${ }^{3}$ followed by interviews with health-care professionals and clinical scientists, ${ }^{4}$ to gather evidence of current recontacting practices. This showed that recontacting does happen in the UK health system, but that there are neither standard procedures between centres, for example, a way of recording patient preferences about recontact, nor agreed events that might trigger a file review and potential recontact. Our findings converge with work on this area, which points to a tension between the ethical desirability of recontacting in some situations, and difficulties with feasibility, supportive infrastructure and resources in practice. $^{2}$ The issue of limited resources is crucial but is compounded with a struggle to define the responsibilities for recontact. For example, we found unclear expectations between clinical scientists in the laboratory and clinicians in relation to whom should keep up-to-date with reclassification of variants. ${ }^{4}$ We also know there can be a clash of expectations between patients and healthcare professionals concerning who should be responsible for recontacting. One study found that clinicians assigned more responsibility for maintaining contact with health-care providers to patients than patients assigned to themselves. ${ }^{5}$

\section{DISCUSSING RECONTACTING WITH PATIENTS}

As a first step to encourage debate, we suggest that recontacting, including issues that might trigger it, should be discussed routinely between patients and clinicians in the context of consent for testing or whenever patient data are collected and recorded. This discussion would help to clarify expectations for clinicians and patients about recontacting in the United Kingdom and elsewhere.

As part of this discussion the patient should be informed that the clinical genetics team holds their records and provides the best information available at the time, but that the patient is welcomed to contact the team: (1) when a potentially relevant family event occurs, such as a death or birth, or a child reaching reproductive age, ${ }^{6}$ and (2) at regular intervals (if agreed by both parties depending on the specific condition).

If the patient agrees, the future contact may also trigger clinicians to review the patient's file to check whether any new information is relevant to them. This idea is in line with a model of shared responsibility (the idea of shared responsibility does not only stem from our empirical research. We are aware that other authors have recently argued for a shared responsibility in relation to disclosure of genetic information to family members $)^{7}$ for recontact with patients, mentioned by some clinicians we interviewed. ${ }^{4}$ It also follows suggestions in previous studies, ${ }^{8,9}$ that involving patients in the process of recontacting could be a solution to practical barriers, as well as ostensibly giving patients more autonomy and control.

If the patient does not agree - for example, if they or the clinician feel they do not have the capacity/ willingness/time to contact the team and/or to be kept up-to-date regarding their condition, the discussion would still help to clarify patient preferences, and the balance of responsibility between patients and clinicians.

The patient would always be able to notify a change of preference to the clinician (this could be in itself a trigger for clinicians to review files).

This discussion and patient preferences should be documented. Having and documenting this discussion with patients could:

1. Reduce a potential clash of expectations between patient and clinicians about responsibility regarding recontacting.

2. Promote patient autonomy - the patient can decide whether to have more or less control over whether recontacting occurs.

3. Respect confidentiality and a right 'not to know', as well as giving patients and their families some control over recontacting in sensitive family situations.

4. Reduce some practical barriers - when patients agree to share responsibility for recontact with clinicians.

5. Promote more standard ways of triggering a file review and potential recontacting event.

\section{FINAL CONSIDERATIONS}

Our proposal is not problem-free. For example, what happens if a patient who agreed to recontact the service for some reason does not? When is the most appropriate time to have the recontacting discussion with patients?

We believe that consultation with stakeholders, including patients, clinical scientists and clinicians, represents an important first step. We are engaging in this work in the 'Recontacting in Mainstreaming Genetics' research project in the United Kingdom (http://ex.ac.uk/ $\mathrm{mgc}$ ). Our intention with this letter is to initiate debate, and we welcome responses. 


\section{CONFLICT OF INTEREST}

The authors declare no conflict of interest.

Daniele Carrieri ${ }^{\star}, 1$, Sandi Dheensa ${ }^{2}$, Shane Doheny ${ }^{3}$, Angus J Clarke ${ }^{3}$, Peter D Turnpenny ${ }^{4}$, Anneke M Lucassen ${ }^{2}$ and Susan E Kelly ${ }^{1}$ ${ }^{1}$ Egenis, University of Exeter, Exeter, UK;

${ }^{2}$ Faculty of Medicine, University of Southampton, Southampton, UK; ${ }^{3}$ School of Medicine, Cardiff University, Cardiff, UK; ${ }^{4}$ Royal Devon and Exeter Hospital, Exeter, UK E-mail:d.carrieri@exeter.ac.uk

1 Levenson D: Legal and ethical issues loom over topic of recontacting patients. Am J Med Genet 2015; 167: vii-viii.
2 Otten E, Plantinga M, Birnie $\mathrm{E}$ et al: Is there a duty to recontact in light of new genetic technologies? A systematic review of the literature. Genet Med 2014, 17 668-678.

3 Carrieri D, Lucassen AM, Clarke AJ et al: Recontact in clinical practice: a survey of clinical genetics services in the United Kingdom. Genet Med 2016; 18: 876-881.

4 Carrieri D, Dheensa S, Doheny S et al: Recontacting in clinical practice: an investigation of the views of healthcare professionals and clinical scientists in the United Kingdom. Eur J Hum Genet 2017; 25: 275-279.

5 Fitzpatrick JL, Han C, Costa T et al: The duty to recontact: attitudes of service providers. Am J Hum Genet 1999; 64: 852-860.

6 Hirschhorn K, Fleisher LD, Godmilow L et al: Duty to re-contact. Policy statement: social ethical and legal issues committee of the American College of Medical Geneticists. Genet Med 1999; 1: 171-172.

7 Wouters RH, Bijlsma RM, Ausems MG et al: Am i my family's keeper? Disclosure dilemmas in next generation sequencing. Hum Mutat 2016; 12: 1257-1262.

8 Almqvist $\mathrm{E}$, Adam $\mathrm{S}$, Bloch $\mathrm{M}$ et al: Risk reversals in predictive testing for Huntington disease. Am J Hum Genet 1997; 61: 945-952.

9 Godard B, Kääriäinen $\mathrm{H}$, Kristoffersson $\mathrm{U}$ et al: Provision of genetic services in Europe: current practices and issues. Eur J Hum Genet 2003; 11: S13-S48. 